\title{
Bocio multinodular gigante. Revisión de la literatura a propósito de un caso
}

\author{
Giant multinodular goiter. Case report and literature review
}

\author{
Roberto J. Causa-Soteras $\mathbb{D}^{\mathbb{D}}$, José R. Causa-García², Yilian A. González-Pérez³ .
}

\begin{abstract}
MD, especialista en Cirugía General; profesor instructor, Hospital Nacional "Simao Mendes", Bissau, Guinea Bissau. MD, especialista en Coloproctología; profesor instructor, Hospital Nacional "Simao Mendes", Bissau, Guinea Bissau. MD, especialista en Ginecología y Obstetricia, profesor instructor, Hospital Nacional "Simao Mendes", Bissau, Guinea Bissau.
\end{abstract}

\section{Resumen}

Introducción. La patología tiroidea abarca desde procesos benignos hasta cánceres. El manejo médico-quirúrgico del bocio gigante implica la realización de estudios citológicos y de imagen. El objetivo de este artículo fue hacer una revisión de la literatura y presentar un caso atendido de bocio multinodular gigante.

Métodos. En el presente artículo se revisa la literatura sobre el tema en cuestión a propósito del caso de una paciente de 54 años de edad, intervenida quirúrgicamente por bocio multinodular, con evolución satisfactoria.

Resultados. Se interviene la paciente de manera electiva, realizando exéresis a un tiroides de 658 gramos, conservando los nervios laríngeos recurrentes y las glándulas paratiroides; se realizó una meticulosa hemostasia y se trasladó la paciente a la Unidad de Cuidados Intensivos. A los 3 días se retiraron ambos drenajes cervicales, y se dio alta hospitalaria definitiva a los 8 días. La biopsia por parafina corroboró el diagnóstico preoperatorio.

Discusión. Las indicaciones de tratamiento quirúrgico del bocio incluyen: crecimiento rápido de la glándula, síntomas compresivos y sospecha de malignidad. Las complicaciones son mínimas cuando se realiza una técnica muy meticulosa.

Conclusión. El bocio multinodular gigante es infrecuente, y en todos los casos, requiere de intervención quirúrgica.

Palabras claves: bocio; bocio nodular; glándula tiroides; tiroidectomía; complicaciones posoperatorias.

Fecha de recibido: 02/09/2019 - Fecha de aceptación: 29/01/2020

Correspondencia: Roberto J. Causa-Soteras, Avenida Pansau Na Isna, Apartado 391 1300, Bissau, Guinea Bissau. Teléfono: +245 $965165533 /+245956582208$

Correo electrónico: causa880326@gmail.com

Citar como: Causa-Soteras RJ, Causa-García JR, González-Pérez YA. Bocio multinodular gigante. Revisión de la literatura a propósito de un caso. Rev Colomb Cir. 2020;35:483-90. https://doi.org/10.30944/20117582.469

Este es un artículo de acceso abierto bajo una Licencia Creative Commons - BY-NC-ND https://creativecommons.org/licenses/by-ncnd/4.0/deed.es 


\begin{abstract}
Introduction. Thyroid pathology ranges from benign processes to cancers. The medical-surgical management of the giant goiter involves performing cytological and imaging studies. The objective of this article was to review the literature and present a treated case of giant multinodular goiter.
\end{abstract}

Methods. This article reviews the literature on the subject in question regarding the case of a 54-year-old female patient who underwent surgery for multinodular goiter, with satisfactory evolution.

Results. The patient was intervened electively, excising a 658 gram thyroid, preserving the recurrent laryngeal nerves and the parathyroid glands. A meticulous hemostasis was performed and the patient was transferred to the Intensive Care Unit. After 3 days, both cervical drains were removed, and the patient was discharged after 8 days. The paraffin biopsy corroborated the preoperative diagnosis.

Discussion. Indications for surgical treatment of goiter include: rapid gland growth, compressive symptoms, and suspected malignancy. Complications are minimal when a very meticulous technique is performed.

Conclusion. Giant multinodular goiter is rare, and in all cases, requires surgical intervention.

Key words: goiter; goiter, nodular; thyroid gland; thyroidectomy; postoperative complications.

\section{Introducción}

La patología tiroidea abarca un amplio abanico de posibilidades, desde procesos benignos hasta cánceres, de comportamientos variables, con función normal de la glándula o alterada, lo que hace laborioso, y a la vez interesante, su estudio y tratamiento.

Desde el punto de vista clínico-semiológico, se define un bocio como "todo aumento de volumen, difuso o localizado, de la glándula tiroides". Desde la óptica anátomo-patológica, solo constituyen bocios aquellas tumoraciones, localizadas o difusas, que se originan por hiperplasia o hipertrofia de los constituyentes normales de la glándula (se excluyen las tiroiditis y las neoplasias) ${ }^{\mathrm{I}}$.

La glándula tiroides normalmente no se ve ni se palpa en el examen físico. El tamaño normal de cada lóbulo tiroideo corresponde aproximadamente (según la OMS) al tamaño de la falange ungueal del pulgar del examinador ${ }^{\mathrm{I}}$.

El manejo médico-quirúrgico del bocio multinodular gigante implica la realización de estudios citológicos y de imagen, con el objetivo de determinar la naturaleza de la lesión y la extensión de esta hacia estructuras vecinas.
Esta patología es infrecuente en la actualidad, y solo presenta elevada prevalencia en zonas con déficit de yodo, generalmente en países africanos, donde los pacientes tienen una dieta inadecuada. Otro factor implicado en el crecimiento de la glándula es el defecto en la síntesis de tiroxina, que aumenta los niveles de la hormona estimulante del tiroides y acelera el crecimiento de las células foliculares ${ }^{2}$.

El bocio multinodular responde poco al tratamiento con tiroxina, por lo tanto, la cirugía es el tratamiento de elección, y es de preferencia, la resección total de la glándula, que generalmente puede removerse por abordaje cervical, aunque el abordaje torácico puede usarse en bocios ectópicos de localización posterior ${ }^{3}$. En pacientes de alto riesgo puede realizarse tratamiento con yodo radioactivo ${ }^{4,5}$.

En el presente artículo se revisa la literatura sobre el tema en cuestión, a propósito del caso de una paciente con diagnóstico de bocio multinodular gigante, atendida en el Hospital Nacional "Simao Mendes", en Guinea Bissau, África Occidental. 


\section{Caso Clínico}

Se trata de una paciente, de 54 años de edad, con antecedentes de hipertensión arterial hace 9 años, que acude a consulta externa de Cirugía General, por presentar aumento de volumen del cuello que, en ocasiones, se asocia con dificultad respiratoria en decúbito supino. En el examen físico se encuentra aumento de volumen en la cara anterior del cuello, no doloroso a la palpación, de bordes bien definidos, con aumento de su vascularización, poco móvil, de consistencia firme, poco adherido a planos profundos, sin soplos, latidos ni expansión. No se palpan adenopatías cervicales ni supraclaviculares (figura I).

En los estudios complementarios encontramos: perfil hormonal tiroideo $\left(\mathrm{T}_{3}, \mathrm{~T}_{4} \mathrm{y} \mathrm{TSH}\right)$, dentro de límites normales; radiografía del tórax, sin alteraciones; ecografía del cuello donde refieren imagen heterogénea que ocupa ambos lóbulos, con predominio del derecho que mide 8Ix64X40 $\mathrm{mm}$ mientras el izquierdo mide $54 \times 33 \times 37 \mathrm{~mm}$, con vascularización periférica, sin adenopatías cervicales visibles; tomografía computarizada (TC) del cuello que muestra aumento de volumen de ambos lóbulos tiroideos, con calcificaciones en su interior, sin extensión al mediastino y sin adenopatías regionales, además de desviación ligera de la tráquea hacia la izquierda. La citología por aspiración con aguja fina (CAAF) descartó la presencia de células neoplásicas e informó como diagnóstico definitivo un bocio multinodular.

Se efectúa el procedimiento indicado de cervicotomía con tiroidectomía total (figura 2). Se identifican y conservan los nervios laríngeos recurrentes y las glándulas parótidas. Se envía la pieza de exéresis quirúrgica (que pesó 658 gramos) a estudio de biopsia por congelación transoperatoria, que corrobora el diagnóstico preoperatorio (figura 3). Se deja drenaje de Penrose en el sitio operatorio, que se exterioriza por contra abertura.

La paciente recibe tratamiento postoperatorio con hidratación parenteral con solución polielectrolítica, analgésicos, antimicrobianos y esteroides a dosis bajas. Se recupera y evoluciona de manera satisfactoria, y se da alta hospitalaria a los 8 días del postoperatorio. En el seguimiento de la paciente, en consulta externa, no se identificaron complicaciones postoperatorias. Se recibe el informe anatomopatológico que corrobora la presencia de bocio multinodular con áreas de calcificación.

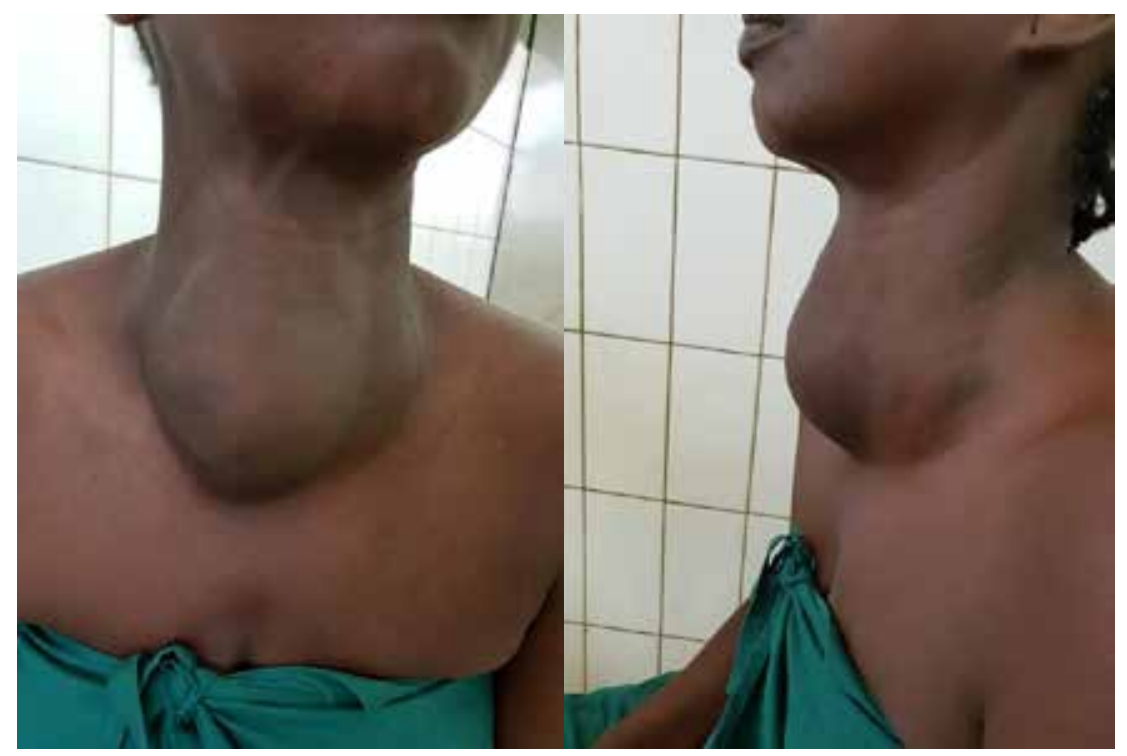

Figura 1. Vista frontal y lateral de la paciente en consulta externa. 


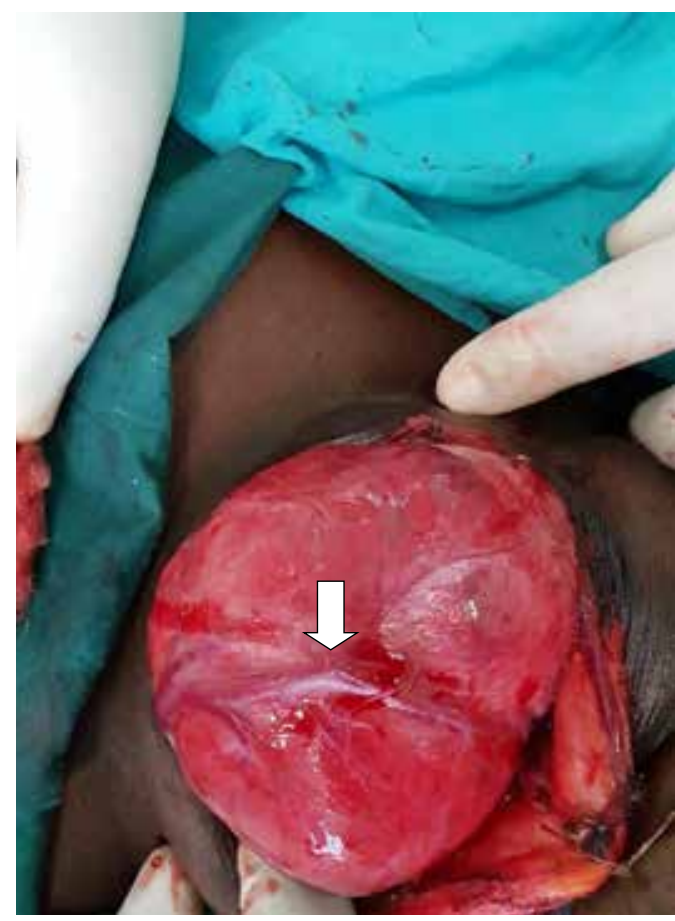

Figura 2. Vista del sitio operatorio. Obsérvese la amplia vascularización de la glándula (flecha blanca).

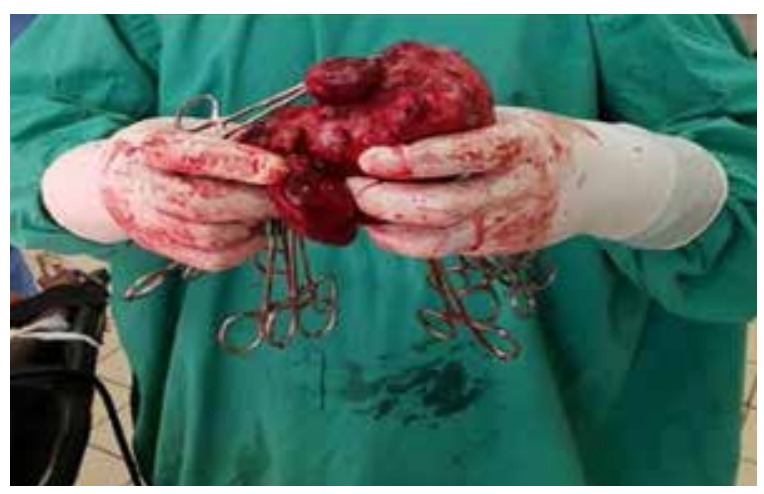

Figura 3. Se muestra la pieza de exéresis quirúrgica.

\section{Revisión de la literatura Fisiopatología}

$\mathrm{Al}$ existir una disminución en los niveles circulantes de hormonas tiroideas (de cualquier etiología), los niveles de hormona tirotropa se incrementan y provocan hipertrofia e hiperplasia de las células foliculares, que dan lugar al aumento de volumen de la glándula tiroidea. Los niveles de $\mathrm{T}_{3} \mathrm{y} \mathrm{T}_{4}$ se pueden normalizar tras el aumento de la hormona estimulante de la tiroides (TSH, por sus siglas en inglés) y desaparecer la hipertrofia e hiperplasia, entonces, se acumula coloide y se origina el bocio coloide ${ }^{4}$.

Ciclos sucesivos de hiperplasia e involución de los folículos dan lugar al bocio multinodular, etapa final de la evolución del bocio simple, el cual puede ser eutiroideo o acompañarse de disfunción tiroidea. Otros factores se involucran en la patogenia del bocio, como: factores de crecimiento epidérmico y de crecimiento similar a la insulina (Inmunoglobulina F), mecanismos inmunitarios (citoquinas, Inmunoglobulina contra receptor de TSH), mutación de oncogenes, inflamación, proliferación o infiltración celular de forma sistémica (leucocitos, histiocitos), o enfermedades metabólicas (amiliodosis) ${ }^{4}$.

En un estudio realizado en el Valle del Cauca, Colombia ${ }^{5}$, se determinó que la deficiencia de yodo puede correlacionarse también con las fuentes de abastecimiento de agua. Se ha encontrado que el agua de un pozo que abastece un distrito con alta incidencia de bocio tiene actividades bociógena y antitiroidea, similares a las producidas por la tioúrea en animales en experimentación. Esta agua produce in vivo aumento de tamaño de la glándula tiroides, depresión de la captación de yodo I3I, baja del contenido de yodo tiroideo y defecto en la formación de diyodotirosina. In vitro rebaja la captación de yodo I3I y la organificación de yodo. Tanto in vivo como in vitro impide el acoplamiento de yodotirosinas, que es necesario para la formación de hormonas tiroideas activas. Los ensayos para aislar e identificar los agentes activos en extractos con diclorometano de agua bociógena han revelado un grupo de hidrocarburos alifáticos saturados y no saturados, algunos de los cuales parecen ser sulfurados. La cromatografía preparativa de gas, usada para determinar cuáles compuestos específicos eran biológicamente activos, reveló tres con marcada actividad antitiroidea, entre los hidrocarburos sulfurados con pesos moleculares por debajo de 220. Al parecer, estos hidrocar- 
buros sulfurados son los bociógenos presentes en el agua, puesto que los agentes antitiroideos más conocidos son compuestos orgánicos que contienen azufre.

Los bociógenos que la contaminan son ampliamente consumidos, por ser el agua un componente tan importante en la dieta. Es por lo tanto probable que, los bociógenos naturales del agua sean el factor etiológico más importante en la endemia de regiones como el Valle del Cauca. Geológicamente estas toxinas sólo podrían derivarse de rocas sedimentarias marinas; formaciones rocosas ricas en hidrocarburos se hallan expuestas esporádicamente en el Valle del Cauca, en las laderas de las cordilleras Central y Occidental. La identificación definitiva de los agentes activos, el esclarecimiento de su origen natural y el conocimiento de sus características biológicas y fisicoquímicas, permitirá desarrollar procedimientos para la inactivación o eliminación de estos compuestos, que puedan ser aplicados a nivel comunitario, para conseguir la erradicación de este importante problema de salud pública.

\section{Formas de presentación del bocio multinodular}

- Asintomático: La mayoría de los casos se desarrollan sin síntomas característicos que puedan ser atribuibles a esta enfermedad. Suele cursar con un perfil tiroideo normal, por lo que el paciente con bocio no debería notar alteraciones de tipo metabólico.

- Síndrome compresivo: Puede dar problemas respiratorios, sobre todo cuando crece hacia el estrecho torácico superior. La valoración clínica de las alteraciones respiratorias producidas al hiperextender el cuello y levantar ambos brazos son una parte importante del examen físico en estos pacientes (maniobras de Kocher y Marañón). En las pruebas de función respiratoria se pueden detectar anomalías sugestivas de obstrucción alta del flujo aéreo. La presencia de disfagia (por compresión del esófago) o disfonía (por compresión nerviosa) son poco frecuentes, pero nos pueden alertar de la necesidad de la extirpación quirúrgica del tiroides.

- Hipertiroidismo: Se estima que el io \% de los casos presentan hipertiroidismo. Entre los síntomas que pueden aparecer encontramos las alteraciones del ritmo cardíaco (arritmias), el aumento del apetito o el insomnio.

- Cáncer de tiroides: Puede aparecer en el contexto de un bocio multinodular después de años de evolución, que ha crecido de forma más evidente en los últimos meses, o en el que han aparecido de forma reciente, síntomas compresivos evidentes.

Los grados de presentación del bocio se muestran resumidos en la tabla $\mathrm{I}$.

\section{Diagnóstico del bocio}

Es necesario sistematizar el estudio de la patología tiroidea para llegar a un diagnóstico preciso y aplicar el tratamiento adecuado, según el estado del paciente y los resultados de los exámenes complementarios efectuados que corroboran el diagnóstico.

En la consulta se deben buscar síntomas que orienten a una deficiencia hormonal o hipotiroidismo, el crecimiento tiroideo, dolor, alteraciones de la voz, procedencia y antecedentes; en el examen físico mediante la palpación se describen las características de la tiroides de forma detallada.

Tabla 1. Grados de presentación del bocio

\begin{tabular}{cl}
\hline Grado & \multicolumn{1}{c}{ Características clínicas. } \\
\hline 0 & No bocio. \\
IA & $\begin{array}{l}\text { Palpable, pero no visible aún con el cuello } \\
\text { hiperextendido. }\end{array}$ \\
IB & $\begin{array}{l}\text { Palpable y visible con el cuello extendido. } \\
\text { No visible con el cuello en posición normal }\end{array}$ \\
2 & Palpable y visible con el cuello en posición normal. \\
3 & Bocio muy voluminoso y visible a distancia.
\end{tabular}


El diagnóstico se apoya con la práctica de diferentes pruebas complementarias, entre los que se encuentran, niveles de calcio, fosforo, fosfatasa alcalina y de función renal, así como la radiografía simple de tórax, donde puede observarse la desviación de la tráquea respecto a la línea media. La tomografía computarizada (TC) ofrece información precisa acerca de posibles relaciones entre los órganos intratorácicos y el bocio, y es de mucha utilidad para el cirujano a la hora de planificar el abordaje quirúrgico ${ }^{6,7}$.

Existen otros estudios que se pueden indicar, como gammagrafía tiroidea y resonancia magnética nuclear, pero no hay mucha experiencia en nuestra región para este estudio del tiroides, ecografía, laringoscopia y $\mathrm{CAAF}^{4}$.

Es de suma importancia el análisis histológico de la pieza (mediante CAAF y biopsia por congelación transoperatoria), pues su resultado definirá el tratamiento y manejo definitivo de los pacientes ${ }^{4,8-\mathrm{I}}$.

\section{Tratamiento}

Para aquellos casos en que el bocio haya sido descubierto de manera casual en el contexto del estudio de otra enfermedad, necesitaremos individualizar el tratamiento del bocio multinodular teniendo en cuenta el contexto del paciente ${ }^{\text {II. }}$

\section{Tratamiento conservador}

$\checkmark$ Levotiroxina en dosis bajas, empezando con 50 ug al día y aumentando gradualmente, vigilando la TSH para evitar una supresión excesiva.

$\checkmark$ Yodo radiactivo: ayuda a disminuir el tamaño de los bocios grandes. La dosis depende del tamaño y de la captación del yodo radiactivo, pero suele ser 3,7 MBq (o,I $\mathrm{mCi})$ por gramo de tiroides corregida en función de la captación (D.H. 370-IO70 MBq (IO-29 mCi)).

$\checkmark \quad$ TSH recombinante a bajas dosis (o,I mg IM).

$\checkmark \quad$ En caso de compresión traqueal aguda va a ser necesario el uso de corticoides (o de cirugía).

\section{Cirugía}

La existencia de un crecimiento rápido de la glándula, la aparición de síntomas compresivos, el hipertiroidismo o la sospecha de malignidad, son las indicaciones que están bien establecidas para el manejo quirúrgico de esta enfermedad; en nuestro caso la cirugía exerética estaba indicada.

En los últimos años se han logrado importantes avances en la cirugía tiroidea ${ }^{12}$, gracias a la creación de diversos instrumentos con el objeto de ayudar al cirujano a mejorar sus resultados en la cirugía tiroidea. Dicha tecnología se ha ido imponiendo en la mayoría de las unidades dedicadas al tratamiento de esta patología, haciendo necesaria la aparición de estudios que evalúen la eficacia y eficiencia de la misma (costo-beneficio) ${ }^{\text {13-I4 }}$. Así, en lo referente a la hemostasia, el bisturí ultrasónico y el sistema de sellado de vasos bipolar, son utilizados en la actualidad por la mayoría de las unidades de cirugía endocrina ${ }^{15,16}$. Los datos publicados indican que el uso del bisturí ultrasónico implica un menor tiempo quirúrgico, siendo controvertidos los datos referentes a costos y tasa de complicaciones ${ }^{17}$.

Por otro lado, respecto a la prevención de las lesiones del nervio laríngeo recurrente, la atención se ha dirigido hacia el uso de instrumentos de monitorización intraoperatoria. Su objetivo es ayudar a preservar la función del nervio mediante su identificación electrofisiológica, sobre todo en casos de grandes bocios, reintervenciones o cirugía de cáncer ${ }^{18,19}$. Han pasado más de 30 años desde que los primeros grupos empezaron a utilizar la monitorización electrofisiológica del nervio recurrente durante la intervención quirúrgica, con resultados dispares en cuanto a prevención de lesiones ${ }^{20,21}$. En los últimos años, vuelve a estar en auge el uso de estos dispositivos. Su uso se basa principalmente en facilitar la identificación del nervio, ayudar en su disección y, lo más importante, pronosticar su función tras la intervención quirúrgica. Respecto a esto último, los autores coinciden en su alto valor predictivo negativo, es decir, su capacidad para predecir función nerviosa postoperatoria normal $(97-99 \%)^{21}$. 
El acceso remoto al cuello es una realidad actual. La idea era esconder la cicatriz en un área no visible, como por ejemplo la axila ${ }^{22}$. De hecho, ya se había planteado la vía axilar para los abordajes mínimamente invasivos directos en el cuello, para extraer las piezas quirúrgicas de cirugía de tiroides demasiado voluminosas. Posteriormente, en lo que llevamos de siglo XXI, se han ido intentando diversos abordajes e instrumentaciones, hasta llegar a las cuatro técnicas de acceso remoto al cuello, con las que existe ya una extensa experiencia a nivel global: el abordaje axilar (con gas o sin gas, robótico o no robótico), el abordaje axilopectoral bilateral (BABA: Bilateral Axillo-Breast Approach), el abordaje retroauricular, y el abordaje transoral -transvestibular (TOETVA: TransOral Endoscopic Thyroidectomy Vestibular Approach) ${ }^{(22)}$. La vía transoral avanza en el abordaje de la tiroides y mejora notablemente el resultado estético del abordaje convencional, siendo un gran impulso en el desarrollo de este tipo de intervención quirúrgica.

\section{Complicaciones}

Las complicaciones que pudieran estar presentes en este tipo de cirugía son variadas. La cirugía es eficaz, pero hay riesgos en ancianos con enfermedad coronaria subclínica ${ }^{3}$.

La incidencia global de lesión del nervio laríngeo recurrente oscila entre 3,12-3,52 \%, siendo más alta en reintervenciones y en cirugía de tumores malignos. Para disminuir esta incidencia, se ha monitorizado el nervio laríngeo recurrente con diferentes métodos ${ }^{23}$. Nuestra paciente no presentó esta complicación, corroborándose la integridad del nervio con su identificación en el transoperatorio. Se realizó laringoscopia directa a la paciente una vez extubada en el quirófano y se observaron las cuerdas vocales en la línea media.

La hemorragia ha sido una de las complicaciones más frecuentes y graves descritas en la tiroidectomía, sobre todo en pacientes con bocio gigante, en la que influye la precisión técnica y otros factores como la hipertensión arterial, tra- tamientos con fármacos antiagregantes y anticoagulantes, la técnica realizada y la enfermedad tiroidea ${ }^{24-26}$. En el caso que presentamos, hubo un adecuado manejo de la hemostasia en el transoperatorio.

El hipoparatiroidismo es otra complicación frecuente, puede ser transitorio, que se presenta en un 2,6 - 5,4\%, o definitivo, en el o,2 y $3 \%{ }^{9}$. En $5 \%$ de pacientes aparece tirotoxicosis autoinmunitaria después del tratamiento. Nuestra paciente no presentó ninguna complicación postquirúrgica, a pesar de lo complejo de la intervención.

\section{Conclusiones}

La tiroidectomía total, en pacientes con bocio multinodular, en que se presentan síntomas de compresión, es un proceder quirúrgico más que justificado y muy seguro, que tiene una tasa de complicaciones mínimas cuando se sigue una evaluación preoperatoria muy exhaustiva y un seguimiento postoperatorio minucioso.

\section{Cumplimiento de normas éticas}

Consentimiento informado: Este estudio es una revisión de la literatura más una revisión retrospectiva de historia clínica, y como tal no hay necesidad de un consentimiento informado ni de aprobación del Comité de Ética Institucional.

Conflicto de interés: Ninguno declarado por los autores.

Fuentes de financiación: Recursos propios de los autores.

\section{Referencias.}

I. Misa-Meliá C, Perna R. Bocio y nódulo tiroideo. Clin Quir Fac Med UdelaR. Uruguay 2019. Fecha de consulta: 20 de enero del 2020. Disponible en: https://www.quirurgicab.hc.edu.uy/images/Bocios_y_n\%C3\%B3dulo_tiroideo_CQFM.pdf

2. Vargas-Uricoechea H, Pinzón-Fernández MV, Bastidas-Sánchez BE. Historia del bocio endémico, desde Sheng-Nung hasta los programas de yodación universal de la sal en Latinoamérica. Rev CES Med. 2018;32:167-77.

3. Iglesias-Díaz G. Bocio intratorácico. Revista de Ciencias Médicas de Pinar del Río. 20I4;I8:IIOI-9. 
4. Parlá-Sardiñas J. Bocio. Rev Cubana Endocrinol. 20I2;23:203-7.

5. Gaitán E. Bociógenos del agua como factor etiológico del bocio endémico en el Valle del Cauca. Colombia médica. 1972;3:136-52. Fecha de consulta: 2I de Enero de 2020. Disponible en: http://colombiamedica.univalle. edu.co/index.php/comedica/article/view/3287

6. Machado-Alba JE, Medina-Morales DA, Valladales-Restrepo LF, Loaiza-Bedoya D, Paredes-Mendoza M. Patrones de prescripción de medicamentos antitiroideos en una población de Colombia. Acta Med Colomb. 20I8;43:I50-5. https://doi.org/I0.36IO4/amc.2018.983

7. Pereira-Despaigne OL, Rodríguez- Fernández Z, Dorimain PC, Falcón- Vilariño GC, Ochoa- Marén G. Diagnóstico de las afecciones nodulares del tiroides. MEDISAN. 2015;19(6):8p. Fecha de consulta: 22 de enero de 2020. Disponible en:

http://medisan.sld.cu/index.php/san/article/view/35I/ pdf_96

8. Herrera F, Redondo K, Osorio C, Grice J, Fernández A. Utilidad de la citología obtenida mediante aspiración con aguja fina en el diagnóstico de las neoplasias foliculares de la glándula tiroides en la E.S.E Hospital Universitario del Caribe: un estudio retrospectivo. Rev Colomb Cir. 2015;30:12-7.

9. Grageda-Soto T, Sandoval J, Huarachi-Loayza M, Grageda-García L, Grageda-García A. Cirugía en patología tiroidea, 20 años de experiencia en el Hospital Elizabeth Seton. Rev Cient Cienc Méd. 2015;18:3I-5.

Io. Díaz-Samada RE, Valdés-Bescosme E, Casin-Rodríguez SM, Reina-Cruz CE, Rodríguez-Hung S. Pacientes operados a causa de enfermedades nodulares de la tiroides. Univ Méd Pinareña. 2019;15:48-56. Fecha de consulta: 22 de enero de 2020. Disponible en:

http:/galeno.pri.sld.cu/index.php/galeno/article/ view/587

II. Breña-Pérez Y, Rosales-Alvarez G, Trasancos-Delgado M, Casanova-Moreno MC, González-Casanova JM. Características clínicas y citológicas en personas con enfermedad nodular tiroidea. Revista de Ciencias Médicas de Pinar del Río. 20I8;22:I0-7.

12. Flores B, Miguel J, Soria V, Moreno A, Carrillo A, Aguayo JL. Eficacia, seguridad y eficiencia de las nuevas tecnologías en cirugía tiroidea. Rev Chil Cir. 20I4;66:320-6. http://dx.doi.org/I0.4067/So718-402620I4000400005

I3. Gómez-Palacios A, Barrios B, Gutiérrez MT, Expósito A, Gómez-Zabala J, Roca B, et al. Morbilidad y costes en las tiroidectomías totales. Mejora del valor del proceso mediante el cambio de gestión. Rev Calid Asist. 20I2;27:I6I-8. https://doi.org/Io.IOI6/j.cali.20II.09.0Io

I4. Iglesias-Díaz G, García-García I, Correa-Martínez L. Características clínico-epidemiológicas de pacientes operados de bocio coloide reintervenidos por recidiva. Medisur. 2015;13:622-9.

15. Lezcano-Bonzi MJ, Adorno A. Frecuencia de patología tiroidea maligna en pacientes tiroidectomizados con diagnóstico de bocio multinodular. Rev. Cir. Parag. 20I8;42:19-23. http://dx.doi.org/IO.I8004/sopaci.20I8.abril.I9-23

I6. Cirocchi R, Boselli C, Guarino S, Sanguinetti A, Tras-tulli S, Desiderio J, et al. Total thyroidectomy with ultrasonic dissector for cancer: multicentric experience. World J Surg Oncol. 20I2;IO:I-5. https://doi.org/IO.II86/I477-7819-IO-70

I7. Lavanderos J, Cacciuttolo G, Cheyre JE. Bocio gigante cérvico-torácico. Rev Chil Cir. 20I4;66:4I4-5.

I8. Mendoza Romo-Ramírez MA., Mendoza-Romo MA. Ramírez-Arriola MC. Cáncer papilar en bocio multinodular gigante. Med. interna Méx. 20I7;33:705-7. https://doi.org/I0.24245/mim.v33i5.1316

19. Osorio C, Fernández A, Ensuncho C, Redondo K, Herrera F. Comparación entre la citología por aspiración con aguja fina y la biopsia por congelación en el diagnóstico de las neoplasias malignas de la glándula tiroides: un estudio prospectivo. Rev Colomb Cir. 2016;31:17-26.

20. Jácome-Roca A. Aportes colombianos a la tiroidología. Revista Colombiana de Endocrinología, Diabetes \& Metabolismo. 20I4;I:33-40.

2I. Oñativia A, Rodríguez-Campoamor N. Tratamiento del bocio endémico con yodo y tiroides. Rev. argent. endocrinol. metab. 2015;52:173-8.

22. Rodríguez-Ramos D, Acevedo-Mejía V, ZapataGonzález R, Bolívar-Mendoza L, Saldarriaga-Henao C. Bocio del mediastino posterior - abordaje mínimamente invasivo: videotoracoscopia derecha más cervicotomía. Rev CES Med. 2016;30:210-6.

23. Martín-Jaramago J, Tamarit-Conejeros M, Escudero-Torrella M, Solaz-Roldán C. Monitorización del nervio laríngeo recurrente mediante tubo orotraqueal electromiográfico en cirugía de tiroides y paratiroides. Consideraciones anestésicas. Rev Esp Anestesiol Reanim. 2013;60:576-83.

24. Pardal-Refoyo J.L. Sistemas de hemostasia en cirugía tiroidea y complicaciones. Acta Otorrinolaringol Esp. 20II;62:339-46.

25. Sosa MG, Ernand RS. Complicaciones derivadas de la tiroidectomía en el Hospital General "Calixto García". Rev Cubana Cir. 20I6;55:27I-8.

26. Martínez-Bello A, Rivera-Real P, Reyes-García MA. Morbilidad posquirúrgica en pacientes sometidos a tiroidectomía en el hospital general de Acapulco. Tres años de experiencia. Cir. gen. 20I4;36:9I-5. 